\title{
Entanglement and nonlocality between disparate solid-state quantum memories mediated by photons
}

\author{
Marcel. li Grimau Puigibert,,${ }^{1,2, *}$ Mohsen Falamarzi Askarani $\odot,{ }^{1,3,}{ }^{*}$ Jacob H. Davidson,,${ }^{1,3}$ Varun B. Verma, ${ }^{4}$ \\ Matthew D. Shaw, ${ }^{5}$ Sae Woo Nam, ${ }^{4}$ Thomas Lutz, ${ }^{1,6}$ Gustavo C. Amaral,,${ }^{1,3,{ }^{*}}$ Daniel Oblak, ${ }^{1}$ and Wolfgang Tittel ${ }^{1,3, \dagger}$ \\ ${ }^{1}$ Institute for Quantum Science and Technology, and Department of Physics \& Astronomy, University of Calgary, \\ 2500 University Drive NW, Calgary, Alberta, Canada T2N 1 N4 \\ ${ }^{2}$ University of Basel, Klingelbergstrasse 82, CH-4056 Basel, Switzerland \\ ${ }^{3}$ QuTech and Kavli Institute of Nanoscience, Delft University of Technology, 2600 GA Delft, The Netherlands \\ ${ }^{4}$ National Institute of Standards and Technology, 325 Broadway, Boulder, Colorado 80305, USA \\ ${ }^{5}$ Jet Propulsion Laboratory, California Institute of Technology, 4800 Oak Grove Drive, Pasadena, California 91109, USA \\ ${ }^{6}$ ETH Zürich, Otto-Stern-Weg 1, 8093 Zürich, Switzerland
}

(Received 20 May 2019; published 13 January 2020)

\begin{abstract}
Entangling quantum systems with different characteristics through the exchange of photons is a prerequisite for building future quantum networks. Proving the presence of entanglement between quantum memories for light working at different wavelengths furthers this goal. Here, we report on a series of experiments with a thulium-doped crystal, serving as a quantum memory for 794-nm photons, an erbium-doped fiber, serving as a quantum memory for telecommunication-wavelength photons at $1535 \mathrm{~nm}$, and a source of photon pairs created via spontaneous parametric down-conversion. Characterizing the photons after re-emission from the two memories, we find nonclassical correlations with a cross-correlation coefficient of $g_{12}^{(2)}=53 \pm 8$; entanglement preserving storage with input-output fidelity of $\mathcal{F}_{I O} \approx 93 \pm 2 \%$; and nonlocality featuring a violation of the Clauser-Horne-Shimony-Holt Bell inequality with $S=2.6 \pm 0.2$. Our proof-of-principle experiment shows that entanglement persists while propagating through different solid-state quantum memories operating at different wavelengths.
\end{abstract}

DOI: 10.1103/PhysRevResearch.2.013039

\section{INTRODUCTION}

Entanglement is central to applications of quantum mechanics [1]. In particular, photon-mediated distribution of entanglement over different and widely spaced quantum systems underpins the creation of a future quantum network [2]. Various materials, devices, and protocols are currently being studied towards this end [3-5], but, so far, there is no certainty about which elements will constitute its fundamental building blocks. However, it appears likely that they will operate within different wavelength regions, ranging from visible [6,7] via near-infrared [8-13], to telecommunication wavelengths $[14,15]$. This will allow leveraging the best properties of each device, and thereby offer heightened capabilities compared to a network consisting of identical quantum systems.

The development of such networks therefore creates a need, immediately and in the future, for hybridization experiments to bridge existing frequency and bandwidth mismatches. One example is linking quantum memories for light

\footnotetext{
*These authors contributed equally to this work

${ }^{\dagger}$ Corresponding author: w.tittel@tudelft.nl
}

Published by the American Physical Society under the terms of the Creative Commons Attribution 4.0 International license. Further distribution of this work must maintain attribution to the author(s) and the published article's title, journal citation, and DOI. operating at different wavelengths through the exchange of photons. However, so far, only a few investigations have been reported [16-18], and none of them has included a quantum memory functioning at telecommunication wavelength. In this work, we demonstrate entanglement between two atomic frequency comb (AFC)-based quantum memories [19] in ensembles of cryogenically cooled rare-earth ions, one for 794-nmand one for 1535 -nm-wavelength photons. The first memory employs a thulium-doped lithium-niobate $\left(\mathrm{Tm}^{3+}: \mathrm{LiNbO}_{3}\right)$ crystal, the second an erbium-doped fiber $\left(\mathrm{Er}^{3+}: \mathrm{SiO}_{2}\right)$. Entanglement is created through the interaction with entangled photons created by spontaneous parametric down-conversion. Both memories allow buffering and re-emitting multiplexed quantum data in feed-forward-controlled spectral or temporal modes, either of which makes them suitable for quantum repeaters [20,21]. It is significant that our experiment involves two classes of ions: Kramers and non-Kramers. Due to their specific electronic configurations, Kramers ions are strongly coupled to the local magnetic environment while nonKramers ions are relatively immune to magnetic-field fluctuations. These characteristics make Kramers ions strong candidates for quantum sensors while non-Kramers are generally more suitable for quantum memory with long storage time.

\section{EXPERIMENTAL SETUP}

Our experimental setup, outlined in Fig. 1 and further detailed in the Appendixes, consisted of four parts: a source 


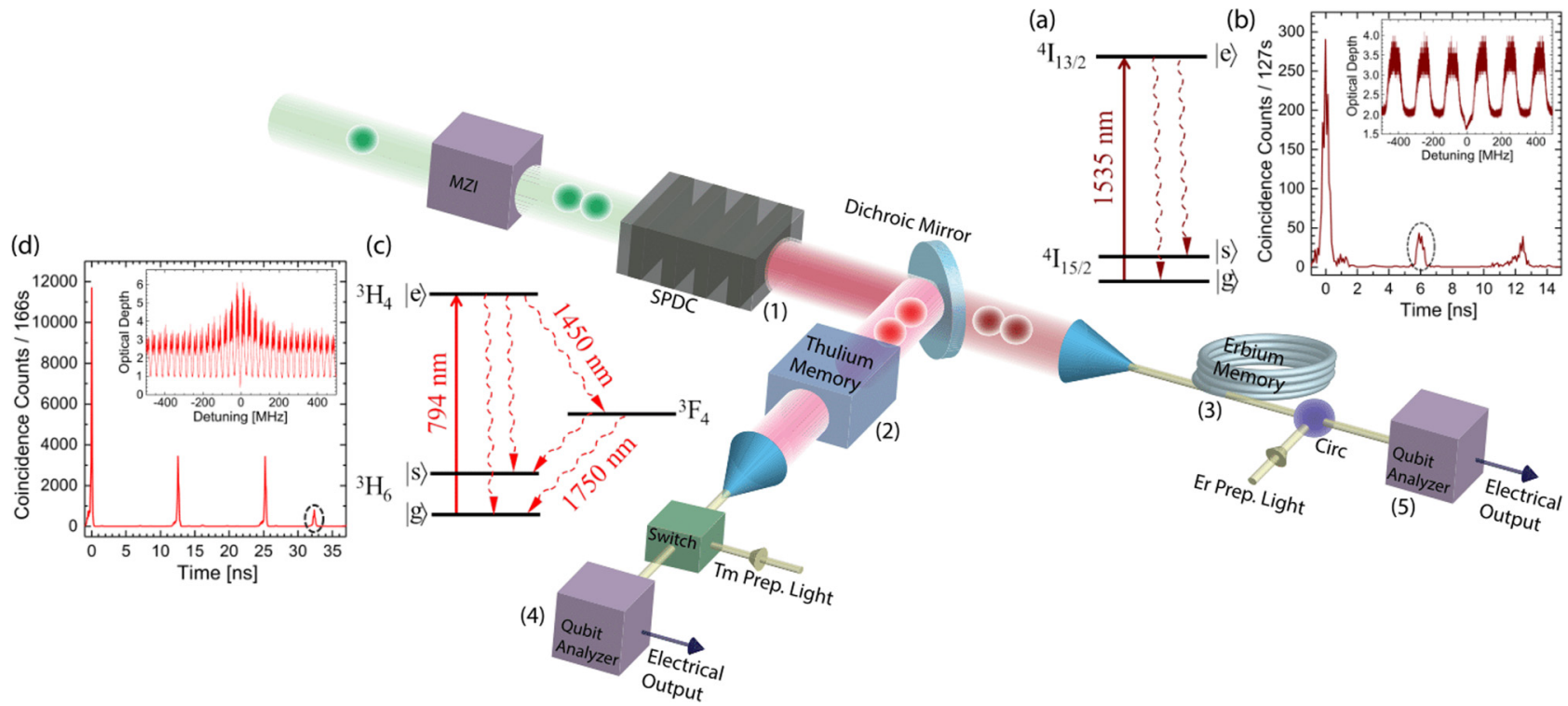

FIG. 1. Photons from a photon-pair source (1) were directed to two different memories $(2,3)$, stored, and re-emitted. Analyzers $(4,5)$ allowed measuring the cross-correlation function, reconstructing density matrices, and testing a Bell inequality. A simplified erbium-level scheme is shown in (a). To create the AFC, erbium ions were frequency-selectively pumped from $|\mathrm{g}\rangle$ via $|\mathrm{e}\rangle$ into the long-lived level $|\mathrm{s}\rangle$. The AFC peak spacing was $\sim 166 \mathrm{MHz}$ (yielding 6 ns storage time) and the total bandwidth was $8 \mathrm{GHz}$ [the inset of (b) shows a 1-GHz-wide section]. Panel (b) shows the storage and retrieval of single photons at $\sim 1535 \mathrm{~nm}$ wavelength in single temporal modes, heralded by the detection of 794-nm photons postselected as coincidences with the reference clock signal (see Appendix D). The coincidence peak at 0 ns is due to directly transmitted (nonstored) photons; the one at $6 \mathrm{~ns}$ (highlighted by a dashed circle) depicts stored and re-emitted photons. Photons created in subsequent pump laser cycles caused accidental coincidences at multiples of $12.5 \mathrm{~ns}$. A simplified thulium level scheme is shown in (c). The AFC preparation was similar to that described for erbium. Equivalent measurements to those in (b) are shown in (d), but now for single photons at $794 \mathrm{~nm}$ heralded by detections of $1535-\mathrm{nm}$ photons again postselected. Note the accidental coincidence peaks at 12.5 and $25 \mathrm{~ns}$. The whole AFC frequency range was $10 \mathrm{GHz}$ (a 1-GHz-wide section is depicted in the inset), and the peak spacing 31 MHz, resulting in 32-ns-long storage.

of entangled photon pairs; two solid-state memories for light (one doped with thulium, and one with erbium); and a detection system comprising analyzers (including detectors), coincidence electronics, and data processing software (see Fig. 2). Appropriate configuration of the source and detection system allowed measuring the cross-correlation function, reconstructing density matrices, and testing Bell inequalities with photon pairs before and after storage.

To create time-bin entangled pairs of photons at 794and $1535-\mathrm{nm}$ wavelengths, short laser pulses at $523 \mathrm{~nm}$ and $80 \mathrm{MHz}$ repetition rate (reference clock) were split by an unbalanced Mach-Zehnder (MZ) interferometer into early $e$ and late $\ell$ temporal modes, and used to pump a nonlinear crystal that is phase-matched for frequency nondegenerate spontaneous parametric down-conversion (SPDC). Assuming the annihilation of exactly one pump photon, the quantum state of the resulting photon pair is described by the so-called $\left|\phi^{+}\right\rangle$Bell state,

$$
\left|\phi^{+}\right\rangle=(|e e\rangle+|\ell \ell\rangle) / \sqrt{2},
$$

where $|x y\rangle \equiv|x\rangle_{794} \otimes|y\rangle_{1535}$, with subscripts denoting each photon's wavelength [22]. The remaining pump light was removed, and the photons of different wavelengths were separated by a dichroic mirror and sent to separate rare-earthion-doped memories. In addition, their spectra were filtered to $8 \mathrm{GHz}$ using an air-spaced etalon (for the $1535-\mathrm{nm}$ photons) and $10 \mathrm{GHz}$ using the inhomogeneously broadened absorption line of Tm (for the 794-nm photons; see Appendix A for details). We adjusted the paths of the photons such that their storage in the memories was temporally overlapped.

The $\mathrm{Tm}^{3+}: \mathrm{LiNbO}_{3}$ crystal, used for storing 794-nm photons, and the $\mathrm{Er}^{3+}: \mathrm{SiO}_{2}$ fiber, used for storing 1535-nm photons, were cooled to $\sim 0.6 \mathrm{~K}$ and exposed to magnetic fields of 125 and $1500 \mathrm{G}$, respectively. Under these conditions it is possible to tailor, by means of optical pumping, the inhomogeneously broadened absorption lines of each ensemble of rare-earth ions into a large number of spectrally equidistant absorption peaks separated by frequency $\Delta$. This feature, known as an atomic frequency comb (AFC), was described as a quantum memory in [19]. After a storage time $\tau=1 / \Delta$ the photon is re-emitted from the memory (for a short theoretical description of the storage protocol see Appendix B). In our experiment, we prepared storage times of 32 and $6 \mathrm{~ns}$, and memory bandwidths of 10 and $8 \mathrm{GHz}$ for the 794- and 1535-nm photons. Note that the short storage times were partly determined by the impossibility to independently optimize the magnetic fields to which the ensembles were exposed in a single cryostat (described in Appendix B).

After re-emission, the photons were directed to analyzers consisting either of a short fiber or an interferometer featuring the same path-length difference as that acting on the pump pulses, and detected using superconducting nanowire singlephoton detectors (SNSPDs) cooled to $0.8 \mathrm{~K}$ in a second cryostat. Finally, the resulting electronic signals were processed in a time-to-digital converter (TDC), and single-detector count 


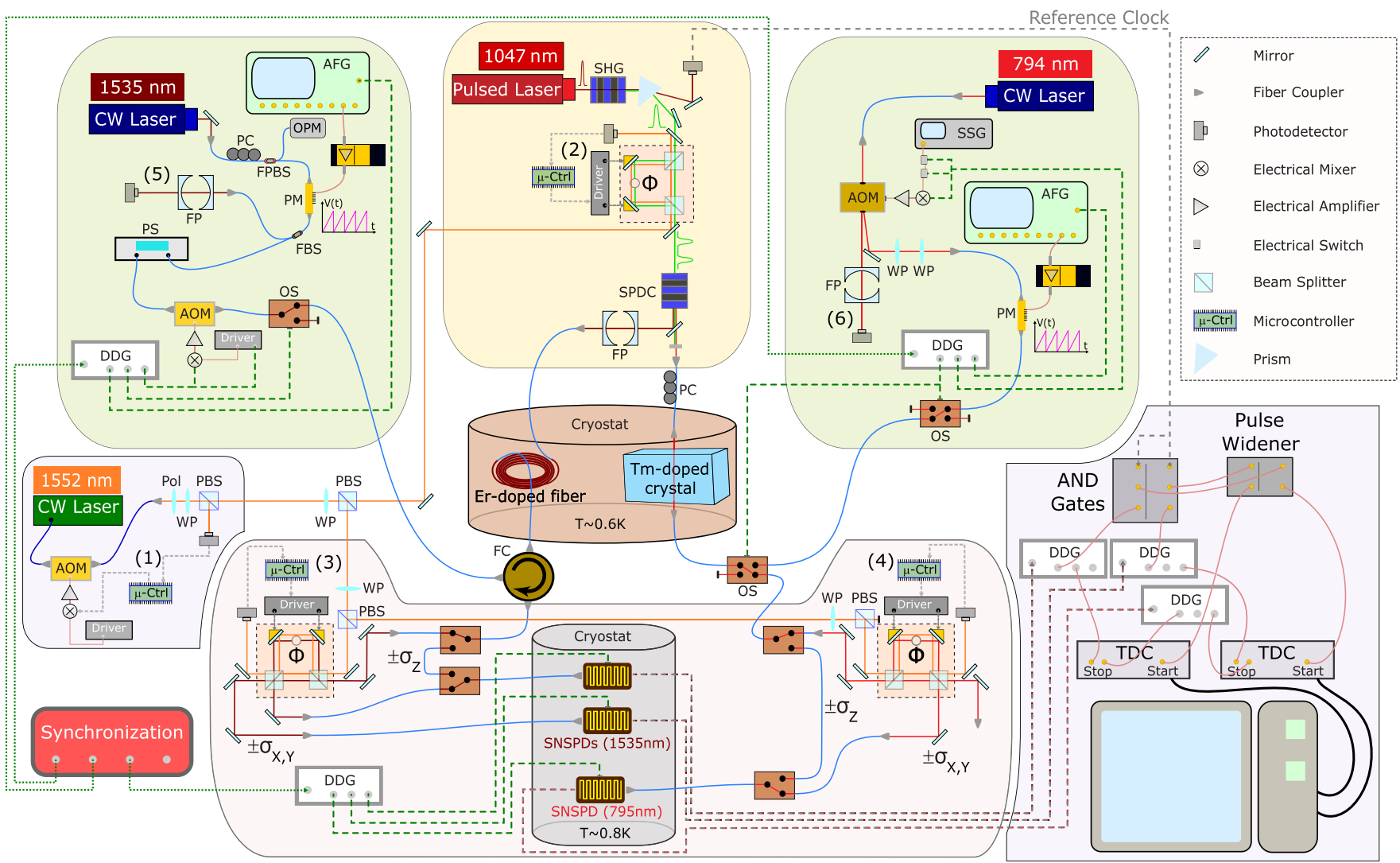

FIG. 2. The figure is divided into six panels that compose the four parts described in the main text. Top left panel: erbium preparation; top center panel: photon pair source; top right panel: thulium preparation; bottom center panel: measurement, analysis and detection; bottom left panel: stabilization source; bottom right panel: electronics and computer software. Red and dark-red lines denote 794- and 1535-nm photons traveling in free space, respectively, and orange lines denote stabilization light. The blue wavy lines depict optical fibers for both wavelengths. All other lines depict electronic cables. SSG: synthesized signal generator. Driver: multipurpose signal driver.

rates as well as time-resolved coincidence count rates were recorded by a computer.

\section{RESULTS}

Initially, we characterized the source and the two memories individually. For this, we blocked the long arm of the pump interferometer such that the source produced pairs of photons in a single temporal mode. We measured the system storage efficiencies for heralded single photons for each memory, finding $0.1 \%$ for the Er-doped fiber and $0.4 \%$ for the Tmdoped crystal. Taking independently characterized input and output coupling efficiencies and transmission loss into account, the corresponding internal efficiencies were $0.5 \%$ and 2\%. See Fig. 1 and Appendix B for more information.

Then, with the long arm of the pump interferometer still blocked, we measured the cross correlation function $g_{12}^{(2)}(\delta t)$ of the photon pairs before storage in the memories (see Appendix D for more information). Creating photons pairs using the highest pump power available, we found $g_{12}^{(2)}(0)=$ $61 \pm 9$, which exceeds the maximum value of 2 that is consistent with the assumption of classical fields [23] by 4.5 standard deviations (see Appendix D for additional measurements). This verifies the nonclassical nature of the photon pairs. Repeating the measurement with memories in place, we found $g_{12}^{(2)}(\delta t=32 \mathrm{~ns}-6 \mathrm{~ns}=26 \mathrm{~ns})=53 \pm 8$ (shown in Fig. 3), where 32 ns and 6 ns denote the preset storage time of the thulium and erbium memories, respectively. This result demonstrates that nonclassical correlations between the members of photon pairs were preserved during storage.

Next, we opened both arms of the pump interferometer, thereby creating the entangled state described in Eq. (1). We used two approaches to establish that the photons remain entangled after storage, and hence that we preserved the entanglement during storage in the distinct quantum memories.

First, we reconstructed the density matrices of the photon pairs before and after storage. This requires measuring a set of joint (bipartite) projectors (see Appendix D). Using a maximum likelihood estimation, the density matrices that best fit the measured projection probabilities were reconstructed; the results are shown in Fig. 4. In turn, this allowed us to calculate the entanglement of formation, the purity, the fidelity of the measured states with the target state $\left|\phi^{+}\right\rangle$in Eq. (1), and the fidelity between input and output states (see Appendix D for more information). These figures of merit, summarized in Table I, confirm that the photons remained entangled.

Finally, we also performed a test of the CHSH Bell inequality [24], which states that the sum of four correlation coefficients $E(\alpha, \beta)$ is upper bounded by 2 if the measurement results can be described by local theories:

$$
S=\left|E\left(\alpha_{1}, \beta_{1}\right)+E\left(\alpha_{1}, \beta_{2}\right)+E\left(\alpha_{2}, \beta_{1}\right)-E\left(\alpha_{2}, \beta_{2}\right)\right| \leqslant 2 .
$$




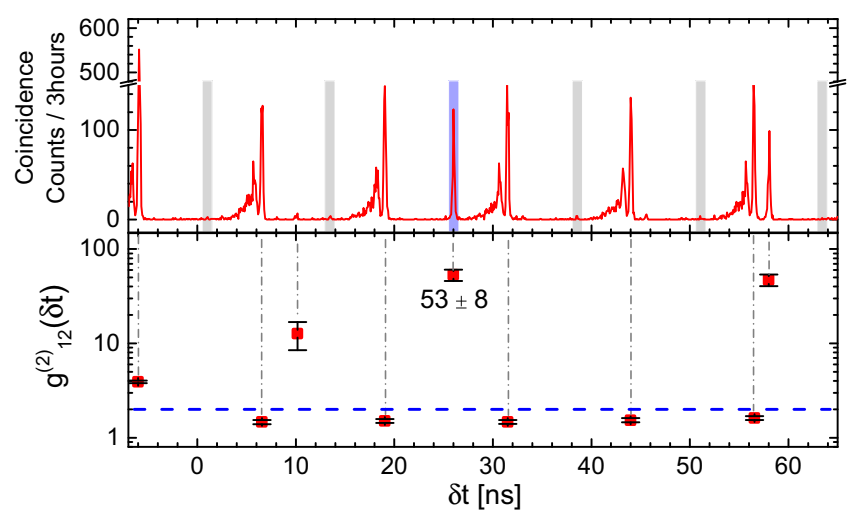

FIG. 3. The upper panel shows the coincidence-detection histogram (within an 80-ps bin width) created by starting the TDC with the clock signal ANDed with re-emitted 1535-nm photon detection signals, and stopping it using all 794-nm photons detected after the Tm memory. In the lower panel, the red squares and the black error bars represent, respectively, $g_{12}^{(2)}(\delta t)$ and uncertainties (one standard deviation), calculated from the upper panel histogram. The blue-dashed line shows the maximum classical value of $g_{12}^{(2)}(\delta t)=2$. Nonclassical correlations between photons after storage can be seen at $\delta t=26 \mathrm{~ns}$. [The rate $R(26 \mathrm{~ns})$ is highlighted using a blue bar, and $R(26 \mathrm{~ns}+n \times 12.5 \mathrm{~ns})$ using gray bars.] The points at $\delta t=58 \mathrm{~ns}$ and $\delta t=10 \mathrm{~ns}$ were caused by imperfections in the thulium AFC (see Appendix B). The data at $\delta t=-6 \mathrm{~ns}$ is due to transmitted (nonstored) 794-nm photons, and data at $\delta t=-6 \mathrm{~ns}+n \times 12.5 \mathrm{~ns}$ corresponds to accidental coincidences with transmitted 794-nm photons emitted in a subsequent pump laser cycle.

Here, $\alpha_{i}$ and $\beta_{i}$ denote different measurement settings—in our case phases set by analyzing interferometers. Using the data given in the Appendix D, we found $S_{\text {in }}=2.52 \pm 0.02$ before, and $S_{\text {out }}=2.6 \pm 0.2$ after storage, both of which significantly exceed 2.

Violating the CHSH Bell inequality (or any other Bell inequality) proves nonlocality rather than entanglement. However, Bell tests allow certifying entanglement within a deviceindependent framework, that is without making assumptions about, e.g., the dimensions of the Hilbert spaces describing the individual quantum systems [25]. (Note that such certification requires the Bell test to be loophole free, while we made the common assumptions of fair sampling and no signaling.) As such, a Bell-inequality violation is a more stringent test of entanglement than finding positive values for the entanglement of formation.

\section{CONCLUSION}

Our results show that entanglement persists while propagating through different solid-state quantum memories operating at different wavelengths. However, for future use in
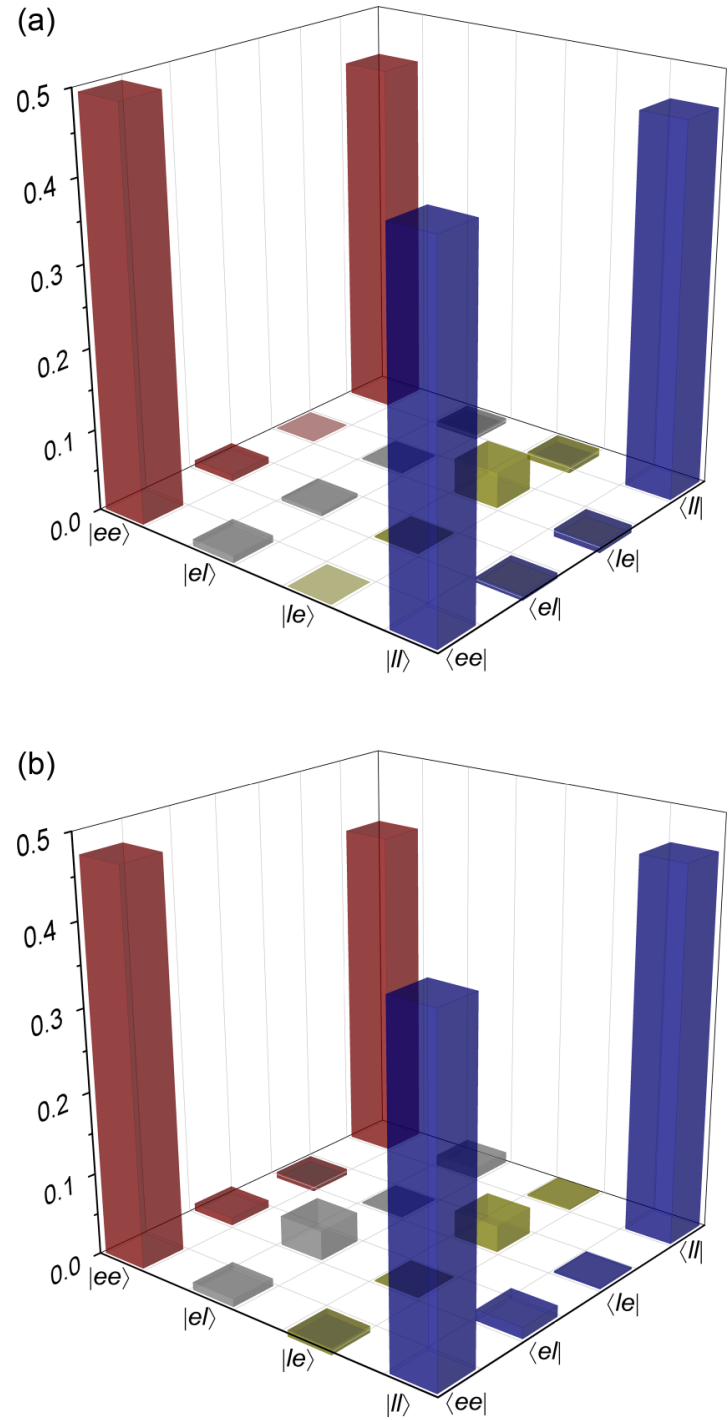

FIG. 4. Reconstructed density matrices of entangled time-bin qubits (a) before and (b) after storage. Only the real parts are shown (the absolute values of all imaginary parts are smaller than 0.023 ).

a quantum network, several factors must be improved. To increase the device efficiency and storage time of the memory for the 794-nm photons, the $\mathrm{Tm}^{3+}: \mathrm{LiNbO}_{3}$ crystal could be replaced by $\mathrm{Tm}^{3+}: \mathrm{Y}_{3} \mathrm{Ga}_{5} \mathrm{O}_{12}$ (Tm:YGG) [26], which features better coherence properties, inside an impedance-matched cavity [27]. Similarly, the erbium-doped fiber could be replaced by a ${ }^{167} \mathrm{Er}$-doped $\mathrm{Y}_{2} \mathrm{SiO}_{5}$ crystal (Er:YSO) [28], again inside a cavity. For more information see Appendix E.

\section{ACKNOWLEDGMENTS}

The authors thank E. Saglamyurek and N. Sinclair for discussions, and acknowledge funding through Alberta

TABLE I. Values were calculated using the density matrices in Fig. 4, and uncertainties estimated using Monte Carlo simulation.

\begin{tabular}{lcccc}
\hline \hline & Entanglement of formation $(\%)$ & Purity $(\%)$ & Fidelity with $\left|\phi^{+}\right\rangle(\%)$ & Input-output fidelity $(\%)$ \\
\hline$\rho_{\text {in }}$ & $81.10 \pm 2.23$ & $84.57 \pm 1.47$ & $91.68 \pm 0.83$ & $93.77 \pm 2.18$ \\
$\rho_{\text {out }}$ & $72.61 \pm 11.70$ & $80.14 \pm 7.22$ & $87.68 \pm 4.67$ & \\
\hline
\end{tabular}


Innovates Technology Futures (AITF), the National Science and Engineering Research Council of Canada (NSERC), and the Netherlands Organization for Scientific Research (NWO). Furthermore, W.T. acknowledges funding as a Senior Fellow of the Canadian Institute for Advanced Research (CIFAR), and V.B.V. and S.W.N. acknowledge partial funding for detector development from the Defense Advanced Research Projects Agency (DARPA) Information in a Photon (InPho) program. Part of the detector research was carried out at the Jet Propulsion Laboratory, California Institute of Technology, under a contract with the National Aeronautics and Space Administration (NASA).

\section{APPENDIX A: TIME-BIN ENTANGLED PHOTON-PAIR SOURCE}

To generate time-bin entangled photon pairs, we employed a 1047-nm wavelength laser (pump laser) emitting 6-ps-long pulses at an $80-\mathrm{MHz}$ repetition rate. A small part of this signal was sent to a photodetector that created the reference clock for all measurements. The remaining portion of the pump laser light was directed to a 2-cm-long periodically poled lithium niobate crystal (PPLN), phase matched for second-harmonic generation (SHG). The then 18-ps-long pulses, centered at $523.5 \mathrm{~nm}$ wavelength, traveled through an unbalanced free-space Mach-Zehnder interferometer (MZI) whose path-length difference corresponded to $1.4 \mathrm{~ns}$ travel time difference, thereby creating pulses of light in early and late temporal modes (or time bins). The emerging pulses were used to pump a second 2-cm-long PPLN crystal, where, with small probability of around $1.6 \%$, spontaneous parametric down-conversion (SPDC) occurred, in which a pump photon is annihilated and a photon pair is created in the maximally entangled state $|\phi\rangle=\frac{1}{\sqrt{2}}(|e e\rangle+|\ell \ell\rangle)$.

As determined by energy conservation and phasematching, the down-converted photons' wavelengths were centered around 794 and $1535 \mathrm{~nm}$-compatible with our quantum memories. Prior to storage, the 1535-nm photons were filtered to $8 \mathrm{GHz}$ bandwidth using an air-spaced FabryPerot cavity (FP) in order to match the bandwidth of the erbium memory. In the case of the 794-nm photons, we took advantage of the fact that the inhomogeneous broadening of $\mathrm{Tm}$ in $\mathrm{LiNbO}_{3}$ extends beyond the 10-GHz-wide AFC: photons outside the AFC bandwidth will be absorbed by Tm ions, eventually be spontaneously re-emitted into random directions, and hence have a negligible probability to reach the detector. This enables restricting the photons' bandwidth without the need for an external filter and thereby reduces complexity and loss. Unfortunately, this approach is not possible in the case of erbium because the fiber guides the absorbed and spontaneously emitted photons (outside the AFC's bandwidth) preferentially towards the detector, thus creating noise.

\section{APPENDIX B: QUANTUM MEMORIES}

\section{Kramers and non-Kramers ions}

When doped into inorganic crystals, rare-earth elements generally form triply positively $(3+)$ charged ions. Ions with and odd and even number of electrons in their $4 f$ orbital are referred to as Kramers and non-Kramers ions, respectively.
$\mathrm{Er}^{3+}$ and $\mathrm{Tm}^{3+}$ are, in this order, examples for these two classes. In the following, we will discuss the principal differences between Kramers and non-Kramers ions, and their impact on the use as quantum memory and quantum transduction.

As they have a half-integer spin, Kramers ions feature an unquenched electronic magnetic moment. When doped into crystals, this results in rapid spin-spin relaxation (spin flip-flops) at small magnetic fields, where the spin levels are equally thermally populated. However, by increasing the magnetic field (and hence the electronic Zeeman level splitting), spin-lattice relaxation becomes dominant due to a rapidly growing phonon density of states. Both relaxation mechanisms shorten the electron spin level lifetime, thereby limiting the efficiency of the optical pumping and, as a consequence, the suitability of Kramers ions at small or moderate magnetic fields for AFC-based quantum memory.

However, at magnetic fields in excess of a few tesla, only the lowest electronic Zeeman level is thermally populated, resulting in the absence of spin flip-flops. As has been shown recently, it is then possible to use long-lived hyperfine levels (arising from the coupling between nuclear and electronic spins) for efficient optical pumping [28].

Note that the high magnetic-field sensitivity of the electronic Zeeman splitting of Kramers ions makes them interesting for optical-to-microwave transduction [29,30] since a transition of a few $\mathrm{GHz}$ - the typical level spacing of superconducting qubits — can be achieved by a small external magnetic field. This benefits coherence times of the closely located superconducting qubits.

Conversely, non-Kramers ions, which do not possess electron spin sublevels owing to a quenched electronic magnetic moment, appear more suitable for photon-echo-based quantum memory due to their long-lived nuclear Zeeman and hyperfine levels. Their usefulness for optical-to-microwave transduction is limited due to the small magnetic-field dependence of their nuclear Zeeman splittings and hence the need for large magnetic fields to achieve a level splitting of a few $\mathrm{GHz}$.

Note that it is reasonable to assume that the fundamental difference between the two types of rare-earth ions affects the sensitivity of a particular ion's spectroscopic properties to host imperfections. Hence, a good host crystal for one ion is not necessarily a good one for an ion from the other class.

\section{AFC protocol}

When a single photon in a well-defined temporal mode is collectively absorbed by ions in an AFC at time $t=0$, the result is a so-called Dicke state of the form

$$
|\psi\rangle_{A}=\frac{1}{\sqrt{N}} \sum_{j=1}^{N} c_{j} e^{-i 2 \pi n_{j} \Delta \cdot t} e^{i k \cdot z}\left|\mathrm{~g}_{1}, \ldots, \mathrm{e}_{j}, \ldots, \mathrm{g}_{N}\right\rangle .
$$

Here, $g$ denotes the atomic ground state and e the excited state, $k$ is the wave number and $z$ the propagation direction of the light, and $j=1 \ldots N$ labels the ions interacting with the photon with weighted amplitudes $c_{j}$ ( $c_{j}$ depends on the ion's detuning $n_{j} \Delta$ with respect to the center frequency of the photon and on its position within the absorbing medium) [19]. 


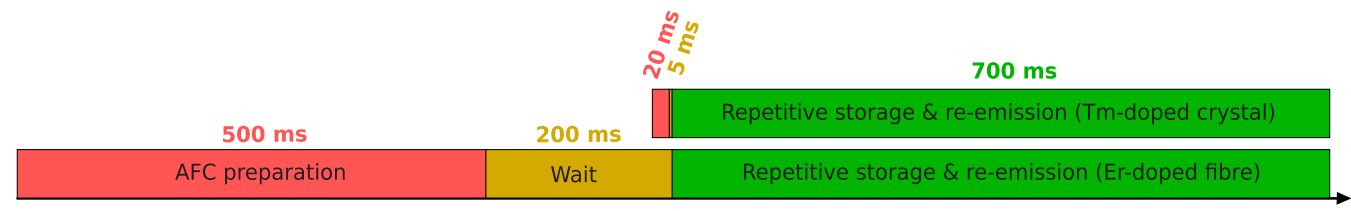

FIG. 5. Timing of experimental sequences in terms of synchronized preparation, wait and storage periods for the two memories.

\section{Erbium doped fiber-AFC preparation}

Continuous-wave telecommunication-wavelength light at $1535 \mathrm{~nm}$ (Er-memory preparation light) was injected from a diode laser into a single-mode optical fiber. A polarization controller (PC) and a fiber-coupled polarization beam splitter (FPBS) with one of its outputs connected to an optical power meter (OPM) allowed controlling the intensity in the second output of the FPBS. The light was then directed to a phase modulator (PM), employed to serrodyne shift its frequency in discrete intervals (each defining a region in which spectral hole-burning took place). The PM was driven by an arbitrary function generator (AFG) connected to an electrical amplifier, allowing for shifts up to $\pm 10 \mathrm{GHz}$.

After the PM, the light went through a polarization scrambler (PS) with a scrambling frequency of $6 \mathrm{kHz}$, used to address all erbium ions despite their randomly oriented transition dipole moments (this is caused by the amorphous $\mathrm{SiO}_{2}$ host). In between the PS and the erbium-doped fiber, an acousto-optic modulator (AOM) was placed. It was driven by 500-ms-long electrical pulses that were synchronized to the master clock. The amplitude of the pulses could be controlled for optimal AFC preparation. In addition, we also inserted a latch-mode optical switch (OS) and a fiber-pigtailed circulator (FC). The OS was connected to port no. 1 of the FC while port no. 2 was connected to the Er-doped fiber, and port no. 3 to the analyzers.

The Er-doped fiber was exposed to a 1500-G magnetic field, which lifts the degeneracy of the electronic ground state and creates two long-lived Zeeman levels with second-long lifetimes.

The interaction between the modulated pump light and the erbium ions leads to frequency-selective persistent spectral hole burning, and, after 1000 repetitions of the 500- $\mu$ s-long burning sequence, to an 8-GHz-wide AFC [see Fig. 1(b) for a 1-GHz-wide central section]. After a wait time of $200 \mathrm{~ms}$, included to ensure the decay of ions from the excited level (avoiding spontaneously emitted photons during the subsequent step), we repeatedly sent, stored (for $6 \mathrm{~ns}$ ), and retrieved members of photon pairs for a total of $700 \mathrm{~ms}$. During this phase of the experimental cycle, the optical switch was toggled to prevent memory preparation light from leaking into the single-photon detectors. See Fig. 5 for a timing diagram.

To read the AFC, port no. 3 of the FC was connected to a photodetector followed by an oscilloscope. After the preparation of the AFC, a reading wave form was applied to the PM, which scanned the laser frequency across the prepared spectral structure from -1 to $+1 \mathrm{GHz}$ while transmission was monitored. To calibrate the measured optical depth (OD), and hence estimate the AFC efficiency, we recorded the transmitted intensity of another 2-ms-long pulse that burned a narrow spectral section to complete transparency.

\section{Thulium-doped lithium niobate crystal-AFC preparation}

Visible-wavelength light at $794 \mathrm{~nm}$ (Tm-memory preparation light) was generated by a diode laser. To obtain a clean spatial mode, the beam was coupled into and out of a singlemode optical fiber. The light was then directed to a single-pass AOM, creating 20-ms-long pulses, and the deflected firstorder beam steered to a set of wave plates for polarization control before being coupled into a fiber-pigtailed PM. Similar to the Er memory preparation, the PM was driven by an AFG and an electrical amplifier for serrodyne shifting. Each sweep took $1 \mathrm{~ms}$ and was repeated 20 times per preparation sequence (see Fig. 5).

After serrodyne shifting in the PM, the light was directed to two fiber-pigtailed optical switches that allowed for routing collimated memory preparation light and single photons in and out of the crystal while avoiding leakage of the classical light into the waiting and storage periods.

The crystal was cooled to $0.6 \mathrm{~K}$ and exposed to a $125-\mathrm{G}$ magnetic field aligned along its $C_{3}$ axis, resulting in two nuclear Zeeman sublevels. Due to their long lifetimes, on the order of minutes, the interaction between the preparation light and the thulium ions leads to persistent spectral holes and allowed creating a 10-GHz-wide AFC [see Fig. 1(d) for a 1-GHz-large central section]. After a waiting time of $5 \mathrm{~ms}$ to avoid spontaneous emission noise, a 700-ms-long storage period was started during which many photons were stored and re-emitted 32 ns later. However, due to spurious modulation of the AFC with periods different from $\Delta=1 / 32 \mathrm{~ns} \sim$ $31 \mathrm{MHz}$, there were two additional moments at which partial rephasing occurred, namely after half and twice the intended storage time $1 / \Delta$ (see Fig. 3 ). This is confirmed by the Fourier transform of the measured AFC structure, shown in Fig. 6.

\section{Optimization of magnetic field}

To optimize an AFC for quantum state storage, it is necessary to carefully set the magnetic field to which an ensemble of rare-earth ions is exposed. In the case of two different ensembles, this implies the need to tune both fields individually. However, in this investigation both fields were created by the same superconducting solenoid (the field difference is due to the ensembles being in different locations with respect to the solenoid), and individual adjustment, and hence optimization of the individual storage efficiencies, was impossible. Instead, we chose a setting that resulted in the best overall efficiency.

More precisely, the erbium-doped fiber requires a magnetic field of around $1500 \mathrm{G}$ to allow for quantum state storage (at other fields spin relaxation prevents efficient optical pumping, as discussed above).

At this setting, the field at the location of the Tm crystal is only around $125 \mathrm{G}$, leading to small nuclear Zeeman splitting. 


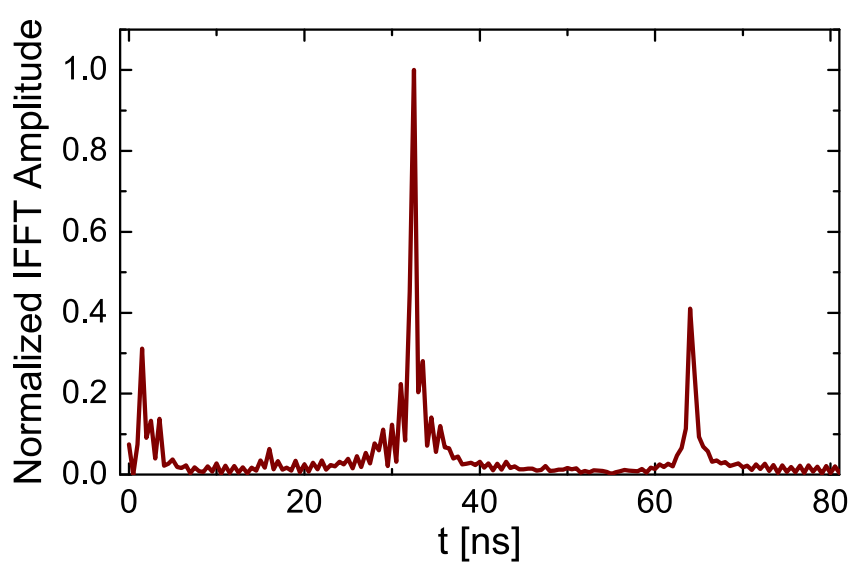

FIG. 6. Inverse Fourier transform of the Tm AFC spectrum [a 1-GHz-broad section is shown in the inset of Fig. 1(d)], showing possible recall times of 16,32 , and $64 \mathrm{~ns}$.

As is always the case if the ground state splitting is smaller than the AFC bandwidth, optical pumping must be tailored to transfer atomic populations from troughs of the AFC to neighboring teeth. Hence, storage time-given by the inverse tooth spacing — and level splitting are not independent anymore. In addition, such cross pumping results in a finesse (the ratio between the teeth spacing and the teeth width) of 2, thereby limiting the storage efficiency [19]. In short, the storage time and the maximum efficiency of the Tm memory are both determined by the need to expose the Er memory to a particular magnetic field. However, since the ground-state electronic Zeeman splitting of erbium exceeds the AFC bandwidth, the storage time in the erbium memory is only limited by the coherence time-it is not determined by the level splitting.

\section{Internal and system efficiencies}

The analysis of the internal efficiencies of the two memories was conducted based on the following two methods. First, by comparing the detection rate of the recalled photons at the output of the cryostat with that of all photons input into the cryostat, we calculated the system efficiency: $\eta_{\text {system }}=$ $R_{\text {out }} / R_{\text {in }}$. In addition to the actual probability for absorbing and re-emitting photons - the internal efficiency-the system efficiency also includes fiber transmission and, for the Tm memory, coupling in and out of the crystal. To estimate the latter two (jointly referred to as $\eta_{\text {coupling }}$ ), we burned a fully transparent spectral hole in the absorption profile of the rare-earth ions using strong light, and then evaluated the ratio of output power over input power: $\eta_{\text {coupling }}=P_{\text {out }} / P_{\text {in }}$. The internal efficiency can then be calculated using $\eta_{\text {device }}=$ $\eta_{\text {system }} / \eta_{\text {coupling. }}$.

In the case of the erbium-doped fiber, the input count rate was $417 \mathrm{~Hz}$, and the rate of retrieved photons behind the cryostat was $1.8 \mathrm{~Hz}$ (both rates were measured in coincidence with the heralding photon). This translates approximately into a $0.1 \%$ system efficiency. Taking into account a $20 \%$ coupling efficiency, we find an internal efficiency of $0.5 \%$. For the thulium-doped crystal quantum memory, identical measurements resulted in $0.4 \%$ for the system and $2.0 \%$ for the internal efficiency.
TABLE II. Efficiency versus wavelength for pairs of wavelengths available in the SPDC source.

\begin{tabular}{lcccc}
\hline \hline$\lambda_{\text {signal }}(\mathrm{nm})$ & $\lambda_{\text {idler }}(\mathrm{nm})$ & $\eta_{\mathrm{TM}}(\%)$ & $\eta_{\mathrm{ER}}(\%)$ & $\eta_{\mathrm{TM}} \times \eta_{\mathrm{ER}}(\%)$ \\
\hline 795.15 & 1534.05 & 0.93 & 0.50 & 0.0095 \\
794.85 & 1535.17 & 1.87 & 0.48 & 0.0182 \\
794.68 & 1535.80 & 2.00 & 0.50 & 0.0200 \\
794.55 & 1565.29 & 1.49 & 0.49 & 0.0148 \\
\hline \hline
\end{tabular}

Alternatively, we estimated the internal efficiency through the analysis of the AFC spectral profile, which we obtained as described in the AFC preparation section of Methods (see Fig. 1 for two examples). The values of the optical depth of the remaining background due to imperfect optical pumping $\left(d_{0}\right)$ and the optical depth of the teeth $\left(d_{1}\right)$, as well as the finesse (F), was inferred by fitting a train of Gaussian functions to the acquired profiles. Then, by using [19]

$$
\eta_{\text {device }}=\left(d_{1} / F\right)^{2} e^{-d_{1} / F} e^{-7 / F_{2}} e^{-d_{0}},
$$

we calculated the internal efficiency. We found $0.5 \%$ for the erbium memory and $2 \%$ for the thulium memory, consistent with the values estimated using the first method.

\section{Optimization of memory wavelengths}

The coincidence count rates in our experiments depend on several interrelated factors, which have to be optimized jointly: the need for energy correlations between the photons belonging to each pair, and the wavelength-dependent storage efficiencies in both memories. To optimize the rate, we measured the device efficiencies as a function of the wavelengths of the two photons produced by the source. The results are presented in Table II.

\section{APPENDIX C: CONTROL AND STABILIZATION}

The experimental setup depicted in Fig. 2 contains many individual elements that must be controlled to ensure physical parameter stability - in particular wavelengths, intensities, and phases-throughout the measurements. Four feedback loops were used, allowing for intensity stabilization of the laser used for phase stabilization of various interferometers [(1) in Fig. 2]; phase stabilization of the pump interferometer [(2) in Fig. 2]; and phase stabilization of the two analyzing interferometers [(3) and (4) in Fig. 2]. Furthermore, to assure that the wavelengths of the lasers used for memory preparation were stable, wavelength control was required [(5) and (6) in Fig. 2].

\section{Intensity stabilization-feedback loop 1}

Continuous-wave light was generated by a fiber-coupled and internally frequency-locked 1532-nm-wavelength laser. After passing through a fiber-pigtailed AOM, used to modulate the light intensity, it was collimated and sent through a bulk-optics polarizer, a wave plate, and a PBS. One output was subsequently used for interferometer stabilization (see below), and the other coupled to a free-space variable-gain photodetector. To stabilize the intensity of the light in the 


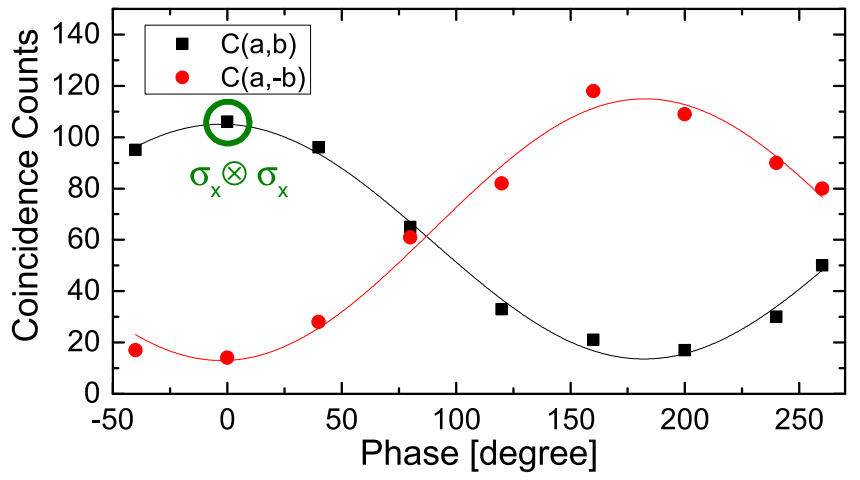

FIG. 7. Visibility curve taken by fixing the phases of the pump and $1535 \mathrm{~nm}$ interferometers to 0 , and sweeping the 794-nm interferometer's piezo voltage, i.e., phase. The $y$ axis shows coincidences between the clock signal, and detections of the re-emitted 794-nm and transmitted 1535-nm photons. The accumulation time per data point was $60 \mathrm{~s}$. The set of phases that define the $\sigma_{x} \otimes \sigma_{x}$ projection corresponds to the point of the curve where $C(a, b)$ is maximum and $C(a,-b)$ minimum.

former output, the detector signal was used as a parameter for the AOM's modulation signal.

\section{Phase stabilization-feedback loops $2-4$}

The frequency- and intensity-stabilized light, split into three individually intensity-controlled beams, was injected into and detected behind each interferometer. Due to interference, any phase variation then caused a variation of the light intensity measured by the corresponding detector. In turn, this allowed locking the interferometers to specific phases using piezomounted mirrors.

Figure 7 displays typical single detection rates (accumulated during $60 \mathrm{~s}$ per data point) in the case where the pump interferometer and the interferometer analyzing the $1535-\mathrm{nm}$ photons were fixed, and the phase of the 794-nm interferometer was swept from $0^{\circ}$ to $360^{\circ}$; the set of phases that corresponds to the $\sigma_{x} \otimes \sigma_{x}$ projector is highlighted.

\section{Laser stability monitoring - circuits 5 and 6}

To ensure wavelength stability of the 1535-nm memory preparation laser, we collected a fraction of the emitted light using a fiber beam splitter (FBS) inserted behind the PM. This light was then directed to a Fabry-Perot (FP) optical cavity, detected by a photodetector, and monitored on an oscilloscope. This allowed us to observe fluctuations of the laser's wavelength. The wavelength of the 794-nm preparation laser was similarly monitored by another FP cavity.

\section{APPENDIX D: DATA COLLECTION AND ANALYSIS}

\section{Photon detection and data collection}

The 794- and 1535-nm photons were detected using tungsten silicide (WSi) superconducting nanowire singlephoton detectors (SNSPDs) cooled inside a sorption fridge to $\sim 0.8 \mathrm{~K}$. Each detector's efficiency was optimized for the wavelength of the detected photons [31]. The average photondetection efficiency, including fiber loss inside the cryostat, was measured to be $70 \%$ for all detectors. The temporal jitter of all detectors was around $250 \mathrm{ps}$, and the dark count rates below $100 \mathrm{~Hz}$. To adjust arrival times of detection signals at the TDC, digital delay generators (DDGs) were placed at the outputs of the SNSPDs (see Fig. 2), and to avoid detections of leaked preparation light, the detectors were only enabled during the 700-ms storage period (see Fig. 5).

As the $80-\mathrm{MHz}$ clock rate from the pulsed pump laser was too high to start the TDC, we instead used a down-sampled version of the clock signal generated by performing an AND gate with the signal from a photon detection event. Adjusting the timing of the signals in the AND gate, we ensured that all nonrelevant start signals, i.e., starts that were not associated with photon pair detection, were discarded. The histogram presented in Fig. 3, which depicts time-dependent detections of photons stored and retrieved from the erbium (thulium) memory, was acquired by starting the TDC with the clock down-sampled by detections of heralding 794-nm (1535-nm) photons generated by the SPDC source. For all other measurements (cross correlation, quantum state tomography, and Bell inequality violation), the TDC was always started by the clock after down-sampling by detections of stored and re-emitted 1535-nm photons.

\section{Acquisition of correlations}

Most of the data are recorded in the form of coincidence counts (per time) or rates. By a coincidence we mean detections in two detectors (labeled 1 for the 1532-nm detector and 2 for the 794-nm detector) that are separated by some time $\delta t$. Let us denote the probability for such a coincidence with detections at time $t$ and $t+\delta t$ as $P_{12}(t ; t+\delta t)$. If we define the time when detector 1 detects a photon to be 0 , we can simplify $P_{12}(0 ; 0+\delta t)=P_{12}(\delta t)$.

In the experimental setup the optical and electronics delays have been aligned such that photons generated as members of the same photon pair will, before storage, be detected in coincidence at $\delta t=0$. The second-order cross-correlation function can be expressed as $g_{12}^{(2)}(\delta t)=P_{12}(\delta t) / P_{1}(0) P_{2}(\delta t)$, where $P_{1}(0)$ and $P_{2}(\delta t)$ denote the probabilities of individual detection at the two times relevant for the coincidence detection. Experimentally, rather than ensuring these individual detection probabilities and the calculation the normalization constant, it is more convenient to extract the product $P_{1}(0) P_{2}(\delta t)$ from the coincidence detection data. To do this we note that the repetition period of our pump laser is $12.5 \mathrm{~ns}$. Moreover, since the photon-pair generation process is spontaneous, there are no statistical correlations between subsequently emitted pairs as has been verified in [32,33]. Hence, the coincidence detection probability $P_{12}(0 ; 12.5 \mathrm{~ns})$ equals the product of the probabilities for individual detections of two uncorrelated photons (i.e., photons from independent pairs emitted $12.5 \mathrm{~ns}$ apart $): P(0 ; 12.5 \mathrm{~ns})=P_{1}(0) P_{2}(12.5 \mathrm{~ns})$.

Such coincidences are commonly referred to as accidental coincidences, and the coincidence rates are identical for any time difference that is an integer multiple of the repetition period, $\delta t=n \times 12.5 \mathrm{~ns}(n \in \mathbb{Z} \neq 0)$. Furthermore, since the probability to detect individual photons is the same for all repetitions, $P_{2}(12.5 \mathrm{~ns})=P_{2}(0)$. It is now easy to see that $g_{12}^{(2)}(0)=P_{12}(\delta t=0) / P_{12}(\delta t=12.5 \mathrm{~ns})$. In other words, we 
can assess the cross-correlation function as the ratio between "true" coincidences and "accidental" coincidences.

The central assumption in our further data analysis is that cross correlations, caused by photons from the source, must obey the same principle regardless the value of $\delta t$, i.e., we can write $g_{12}^{(2)}(\delta t)=P_{12}(\delta t) / P_{12}(\delta t+12.5 \mathrm{~ns})$. This allows us to express $g_{12}^{(2)}(\delta t)$ as a ratio of coincidence count rates [23],

$$
g_{12}^{(2)}(\delta t)=R(\delta t) /\langle R(\delta t+12.5 \mathrm{~ns})\rangle_{\mathrm{n}},
$$

where $P_{12}(\delta t)=\alpha R(\delta t)$ with a proportionality factor $\alpha$ that cancels in $g_{12}^{(2)}$, and " $\langle\ldots\rangle_{\mathrm{n}}$ " denotes averaging over several repetition periods $n$, in order to reduce the statistical uncertainty of the measurement. Specifically, we chose $n=\{[-5,-1],[1,5]\}$.

Figure 3 depicts cross-correlation functions for different $\delta t$, calculated according to Eq. (D1). For this measurement we align the timing such that, without storage, the transmitted photons are in coincidence for $\delta t=0$. With storage, however, we herald using detections of $1535-n m$ photons that have been stored for $6 \mathrm{~ns}$. Hence, we expect the nonabsorbed 795-nm photons to be in coincidence at $\delta t=-6 \mathrm{~ns}$, with accidental coincidences at $\delta t=(-6+n \times 12.5) \mathrm{ns}$. The stored and recalled 795-nm photons will, on the other hand, give rise to coincidences at $\delta t=(32-6) \mathrm{ns}=26 \mathrm{~ns}$, with accidental coincidences at $\delta t=(26+n \times 12.5) \mathrm{ns}$. Figure 3 shows that the $g_{12}^{(2)}$ only exceeds the classical bound at the two aforementioned delays as well as at $\delta t=10 \mathrm{~ns}$ and $\delta t=56 \mathrm{~ns}$, which are erroneously re-emitted photons due to AFC imperfections (see Fig. 6). An important observation is that even if the accidental coincidence peaks are large, the corresponding cross correlation remains well below the classical bound, reflecting independent (nonquantum) nature of the detections.

Another interesting feature is that the cross correlation after storage is significantly larger $\left[g_{12}^{(2)}(26 \mathrm{~ns})=53 \pm 8\right]$ than before storage $\left[g_{12}^{(2)}(-6 \mathrm{~ns})=3.9 \pm 0.1\right]$. To understand this, let us point out that, in order to minimize loss, we decided to spectrally filter the 794-nm photons only using the AFC memory, and not to put any filter cavity directly behind the source (as in the case of the 1535-nm photons). Hence, coincidences between filtered heralding photons at $1535 \mathrm{~nm}$ and unfiltered 794-nm photons (before storage) will contain significant contributions from uncorrelated 794-nm photons outside the AFC bandwidth. However, these contributions are eliminated when measuring coincidences with recalled 794-nm photons because these photons must be spectrally overlapped with the AFC and, thus, match the spectrum of the heralding photons. This explanation is supported by the data in Fig. 8 for the values of $g_{12}^{(2)}(\delta t)$ for the source (no storage) and for the stored and re-emitted photons, both as a function of the pump power. For this measurement, the cross correlation for the source was measured with a matching FP etalon in the 794-nm photon arm and, hence, only true coincidences were recorded. In this case, the $g_{12}^{(2)}(0)$ for the non-stored photons agrees within the experimental uncertainty with that of the stored photons. Note that this measurement was taken at the highest pump power, which was also used for the $g_{12}^{(2)}(0)$ measurement shown in Fig. 3.

The $g_{12}^{(2)}(0)$ values for the stored photons in Fig. 8 also highlight the influence of background noise in our measure-

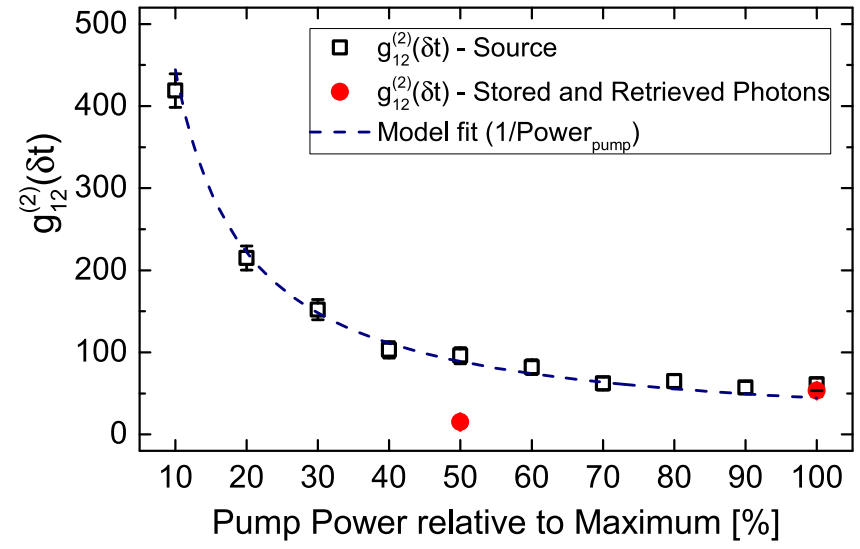

FIG. 8. Measured $g_{12}^{(2)}(\delta t)$ for different values of the pump power. As expected, the value of $g_{12}^{(2)}(\delta t)$ for the source increases with decreasing pump power. However, to achieve a higher SNR, we used the highest available pump power throughout all measurements involving quantum memories. The fit function is derived from a first-order approximation of the SPDC Hamiltonian, i.e., $g_{12}^{(2)}(\delta t) \propto \frac{1}{\text { Powerpump }}$.

ment. Without storage, $g_{12}^{(2)}(0)$ follows the expected inverse dependence on pump power-the smaller the pump power the larger the $g_{12}^{(2)}(0)$. For the stored photons, however, the trend is oppositive, and $g_{12}^{(2)}(0)$ decreases as the pump power is reduced. This is because the coincidence rate after storage is small enough such that the background noise starts to dominate.

\section{Qubit analysis}

Free-space interferometers with path-length differences identical to that of the pump interferometer and controlled relative phases were used to project individual qubits of the entangled state onto superposition states $\frac{1}{\sqrt{2}}\left(|e\rangle+e^{i \theta}|\ell\rangle\right.$, where the phase $\theta$ was established as described in Fig. 7. Apart from the active phase stabilization described above, the phase of the interferometers was passively stabilized by temperature controlling its enclosure. Alternatively, to measure qubits in the canonical bases, i.e., $|e\rangle$ and $|\ell\rangle$, the interferometers were replaced by a short fiber, and the photon arrival time was recorded.

To reconstruct two-photon density matrices, we measured several bipartite projectors composed of individual projections onto eigenstates of combinations of the Pauli operators $\sigma_{x}, \sigma_{y}$, and $\sigma_{z}$. In the case of temporal mode encoding, this is done by means of interferometers with suitably chosen phases (for $\sigma_{x}$ and $\sigma_{y}$ ), or delay lines (for $\sigma_{z}$ ), respectively.

\section{Entanglement of formation, purity, and fidelity calculation}

The entanglement of formation is defined as

$$
E_{F}(\rho)=H\left(0.5+0.5 \sqrt{1-C^{2}(\rho)}\right),
$$

where $H(x)=-x \log _{2}(x)-(1-x) \log _{2}(1-x)$ and $C(\rho)$ is the concurrence, which is defined as

$$
C(\rho)=\max \left\{0, \lambda_{1}-\lambda_{2}-\lambda_{3}-\lambda_{4}\right\} .
$$


TABLE III. Joint-detection probabilities used for the reconstruction of the density matrices before (in) and after (out) storage.

\begin{tabular}{lccccc}
\hline \hline & $\sigma_{z} \otimes \sigma_{z}$ & $\sigma_{z} \otimes \sigma_{x}$ & $\sigma_{z} \otimes \sigma_{y}$ & $\sigma_{x} \otimes \sigma_{z}$ & $\sigma_{x} \otimes\left(\sigma_{x}+\sigma_{y}\right)$ \\
\hline$P_{\text {in }} \%$ & $49.55 \pm 2.48$ & $25.51 \pm 0.43$ & $24.96 \pm 0.43$ & $26.85 \pm 0.41$ & $41.72 \pm 1.08$ \\
$P_{\text {out }} \%$ & $48.21 \pm 2.48$ & $25.00 \pm 5.89$ & $24.49 \pm 7.07$ & $25.00 \pm 5.10$ & $40.91 \pm 6.82$ \\
& $\sigma_{x} \otimes \sigma_{y}$ & $\sigma_{y} \otimes \sigma_{z}$ & $\sigma_{y} \otimes \sigma_{x}$ & $\sigma_{y} \otimes\left(\sigma_{x}+\sigma_{y}\right)$ & $\sigma_{y} \otimes\left(\sigma_{x}-\sigma_{y}\right)$ \\
$P_{\text {in }} \%$ & $23.82 \pm 0.57$ & $27.15 \pm 0.42$ & $25.22 \pm 0.59$ & $8.66 \pm 0.58$ & $40.39 \pm 1.05$ \\
$P_{\text {out }} \%$ & $22.22 \pm 5.56$ & $25.26 \pm 5.16$ & $26.39 \pm 6.05$ & $6.82 \pm 3.94$ & $40.00 \pm 8.16$ \\
& $\sigma_{z} \otimes \sigma_{-z}$ & $\sigma_{z} \otimes \sigma_{-x}$ & $\sigma_{z} \otimes \sigma_{-y}$ & $\sigma_{x} \otimes \sigma_{-z}$ & $\sigma_{x} \otimes\left(\sigma_{-x}+\sigma_{-y}\right)$ \\
$P_{\text {in }} \%$ & $2.26 \pm 0.10$ & $23.37 \pm 0.42$ & $23.63 \pm 0.41$ & $20.81 \pm 0.36$ & $9.04 \pm 0.50$ \\
$P_{\text {out }} \%$ & $2.30 \pm 0.54$ & $26.39 \pm 6.05$ & $26.53 \pm 7.36$ & $27.08 \pm 5.31$ & $9.09 \pm 3.21$ \\
& $\sigma_{x} \otimes \sigma_{-y}$ & $\sigma_{y} \otimes \sigma_{-z}$ & $\sigma_{y} \otimes \sigma_{-x}$ & $\sigma_{y} \otimes\left(\sigma_{-x}+\sigma_{-y}\right)$ & $\sigma_{y} \otimes\left(\sigma_{-x}-\sigma_{-y}\right)$ \\
$P_{\text {in }} \%$ & $26.18 \pm 0.60$ & $20.48 \pm 0.36$ & $24.78 \pm 0.59$ & $41.34 \pm 1.26$ & $9.61 \pm 0.51$ \\
$P_{\text {out }} \%$ & $27.78 \pm 6.21$ & $24.21 \pm 5.05$ & $23.61 \pm 5.73$ & $43.18 \pm 9.91$ & $10.00 \pm 4.08$ \\
& $\sigma_{-z} \otimes \sigma_{z}$ & $\sigma_{-z} \otimes \sigma_{x}$ & $\sigma_{-z} \otimes \sigma_{y}$ & $\sigma_{-x} \otimes \sigma_{z}$ & $\sigma_{-y} \otimes \sigma_{z}$ \\
$P_{\text {in }} \%$ & $2.15 \pm 0.11$ & $24.45 \pm 0.43$ & $24.74 \pm 0.42$ & $28.16 \pm 0.42$ & $28.54 \pm 0.43$ \\
$P_{\text {out }} \%$ & $2.17 \pm 0.53$ & $23.61 \pm 5.73$ & $24.49 \pm 7.07$ & $26.04 \pm 5.21$ & $25.26 \pm 5.16$ \\
& $\sigma_{-z} \otimes \sigma_{-z}$ & $\sigma_{-z} \otimes \sigma_{-x}$ & $\sigma_{-z} \otimes \sigma_{-y}$ & $\sigma_{-x} \otimes \sigma_{-z}$ & $\sigma_{-y} \otimes \sigma_{-z}$ \\
$P_{\text {in }} \%$ & $46.03 \pm 0.41$ & $26.66 \pm 0.44$ & $26.67 \pm 0.44$ & $24.18 \pm 0.39$ & $23.83 \pm 0.39$ \\
$P_{\text {out }} \%$ & $47.31 \pm 2.46$ & $25.00 \pm 5.89$ & $24.49 \pm 7.07$ & $21.88 \pm 4.77$ & $25.26 \pm 5.16$ \\
\hline \hline
\end{tabular}

The $\lambda_{i}$ 's are the eigenvalues of the reconstructed density matrix shown in Fig. 4.

The fidelity between $\rho$ and $\sigma$ is

$$
F(\rho, \sigma)=(\operatorname{Tr}(\sqrt{\sqrt{\rho} \sigma \sqrt{\rho}}))^{2}
$$

and the purity of a state $\rho$ is

$$
P=\operatorname{Tr}\left(\rho^{2}\right) .
$$

\section{Bell-inequality test}

The four correlation coefficients that compose $S$ in Eq. (2) were measured as

$$
E(a, b)=\frac{C(a, b)-C(a,-b)}{C(a, b)+C(a,-b)}
$$

using projectors $a=\sigma_{x}, a^{\prime}=\sigma_{y}, b=\left(\sigma_{x}+\sigma_{y}\right)$, and $b^{\prime}=$ $\left(\sigma_{x}-\sigma_{y}\right)$. As explained in more detail in [11], these projections correspond to detecting photons in specific outputs of interferometers with appropriately chosen phases. Tables III and IV present the individual values acquired for quantum state tomography and CHSH-Bell inequality tests, respectively.

\section{APPENDIX E: FUTURE IMPROVEMENTS}

In order to meet the benchmarks imposed by a spectrally multiplexed quantum repeater [20], the storage time and storage efficiency have to be significantly improved for both the $\mathrm{Tm}$ - as well as the Er-doped memory.
The simplest implementation that allows one to achieve in principle unit storage efficiency is the use of an impedancematched cavity [27] in which the reflectivity of the front mirror is determined by the round-trip loss in the rare-earthion doped crystal (the back mirror is assumed to be $100 \%$ reflective). This idea has very rapidly enabled increasing the quantum memory efficiency from a few percent to $56 \%$ [34]. In addition, the cavity also restricts the AFC bandwidth to the cavity linewidth, and spectral modes to resonances spaced by the cavity free-spectral range. Calculations based on reasonable parameters for crystal length (e.g., $1 \mathrm{~mm}$ ) and optical depth (e.g., 2) result in an AFC width on the order of $1 \mathrm{GHz}$ and memory channels spaced by around $5 \mathrm{GHz}$. These are values that allow the use of our current photon pair source without modification.

The optical coherence time- the fundamental limit to the storage in optical coherence-of the 795-nm transition in $\mathrm{Tm}: \mathrm{Y}_{3} \mathrm{Ga}_{5} \mathrm{O}_{12}$ (Tm:YGG) at $1.2 \mathrm{~K}$ approaches $500 \mu \mathrm{s}$, a factor of 15 more than in $\mathrm{Tm}: \mathrm{LiNbO}_{3}$ at the same temperature, with further improvements expected at lower temperature [26]. Paired with long-lived nuclear Zeeman levels, allowing for efficient optical pumping, and a simple sublevel structure, this makes this crystal a very promising memory candidate for a quantum repeater architecture based on spectral multiplexing [20]. In particular, when combined with an impedancematched cavity, quantum state storage with $90 \%$ efficiency during a time on the order of $100 \mu \mathrm{sec}$-sufficient for an elementary link length in excess of $20 \mathrm{~km}$-can be expected.

TABLE IV. Correlation coefficients used to test the CHSH-Bell inequality.

\begin{tabular}{lcccc}
\hline \hline & $a \otimes b$ & $a \otimes b^{\prime}$ & $a^{\prime} \otimes b$ & $a^{\prime} \otimes b^{\prime}$ \\
\hline$E_{\text {in }} \%$ & $60.59 \pm 1.34$ & $64.39 \pm 1.27$ & $-61.56 \pm 1.31$ & $65.40 \pm 1.48$ \\
$E_{\text {out }} \%$ & $68.73 \pm 11.29$ & $63.03 \pm 8.22$ & $-59.28 \pm 10.32$ & $68.07 \pm 10.34$ \\
\hline \hline
\end{tabular}


Er-doped $\mathrm{Y}_{2} \mathrm{SiO}_{5}$ (Er:YSO) features the longest optical coherence time of an impurity in a solid-state material, around $4.4 \mathrm{~ms}$ [35]. This is an improvement by more than a factor of $10^{4}$ compared to $\mathrm{Er}^{\mathrm{SiO}} \mathrm{S}_{2}$. However, as mentioned in the Kramers and non-Kramers ions section of Methods, due to significant spin-spin and spin-lattice relaxation, a quantum memory has so far not been demonstrated in this crystal. But it has very recently been discovered that the application of a 6-T magnetic field across a ${ }^{167} \mathrm{Er} \mathrm{Y}_{2} \mathrm{SiO}_{5}$ crystal results in a hyperfine sublevel lifetime exceeding 1 min and an optical pumping efficiency of 95\%, making it very promising for storage of telecommunication wavelength photons [28]. Hence, quantum state storage with $90 \%$ efficiency during a ms using optically excited coherence should be possible. Moreover, instead of increasing the hyperfine sublevel lifetime in order to make AFC-based quantum memories possible, one could also reduce the excited level lifetime by means of the Purcell effect in a nanocavity [36].

Note that by replacing the currently used host materials $\mathrm{LiNbO}_{3}$ and $\mathrm{SiO}_{2}$ by YGG and YSO, no significant changes to the preparation procedure of the quantum memories are necessary. Furthermore, adding an impedance-matched cavity to the memory adds the requirement for proper mode matching, which, however, constitutes no significant technical problem. It should also be noted that the spectral multiplexing scheme proposed in [20] does not rely on on-demand recall-re-emission after a predetermined storage time (given by the inverse AFC teeth spacing) is sufficient. In short, our proof-of-principle demonstration can readily be translated into workable quantum technology by employing optimized host materials and suitable cavities.
[1] R. Horodecki, P. Horodecki, M. Horodecki, and K. Horodecki, Quantum entanglement, Rev. Mod. Phys. 81, 865 (2009).

[2] S. Wehner, D. Elkouss, and R. Hanson, Quantum internet: A vision for the road ahead, Science 362, eaam9288 (2018).

[3] A. Lvovsky, B. C. Sanders, and W. Tittel, Optical quantum memory, Nat. Photon. 3, 706 (2009).

[4] M. D. Eisaman, J. Fan, A. Migdall, and S. V. Polyakov, Invited review article: Single-photon sources and detectors, Rev. Sci. Instrum. 82, 071101 (2011).

[5] N. Somaschi, V. Giesz, L. De Santis, J. C. Loredo, M. P. Almeida, G. Hornecker, S. L. Portalupi, T. Grange, C. Antón, J. Demory et al., Near-optimal single-photon sources in the solid state, Nat. Photon. 10, 340 (2016).

[6] B. Hensen, H. Bernien, A. E. Dréau, A. Reiserer, N. Kalb, M. S. Blok, J. Ruitenberg, R. F. L. Vermeulen, R. N. Schouten, C. Abellán et al., Loophole-free bell inequality violation using electron spins separated by 1.3 kilometres, Nature (London) 526, 682 (2015).

[7] M. P. Hedges, J. J. Longdell, Y. Li, and M. Sellars, Efficient quantum memory for light, Nature (London) 465, 1052 (2010).

[8] L. Li, Y. Dudin, and A. Kuzmich, Entanglement between light and an optical atomic excitation, Nature (London) 498, 466 (2013).

[9] Y. Yu, F. Ma, X.-Y. Luo, B. Jing, P.-F. Sun, R.-Z. Fang, C.-W. Yang, H. Liu, M.-Y. Zheng, X.-P. Xie et al., Entanglement of two quantum memories via metropolitan-scale fibers, arXiv:1903.11284.

[10] V. Krutyanskiy, M. Meraner, J. Schupp, V. Krcmarsky, H. Hainzer, and B. P. Lanyon, Light-matter entanglement over $50 \mathrm{~km}$ of optical fibre, npj Quantum Inf. 5, 72 (2019).

[11] E. Saglamyurek, N. Sinclair, J. Jin, J. A. Slater, D. Oblak, F. Bussières, M. George, R. Ricken, W. Sohler, and W. Tittel, Broadband waveguide quantum memory for entangled photons, Nature (London) 469, 512 (2011).

[12] C. Clausen et al., Quantum storage of photonic entanglement in a crystal, Nature (London) 469, 508 (2011).

[13] L. Hanschke, K. Fischer, S. Appel, D. Lukin, J. Wierzbowski, S. Sun, R. Trivedi, J. Vučković, J. Finley, and K. Müller, Quantum dot single-photon sources with ultra-low multi-photon probability, npj Quantum Inf. 4, 43 (2018).
[14] E. Saglamyurek, J. Jin, V. B. Verma, M. D. Shaw, F. Marsili, S. W. Nam, D. Oblak, and W. Tittel, Quantum storage of entangled telecom-wavelength photons in an erbium-doped optical fibre, Nat. Photon. 9, 83 (2015).

[15] J.-H. Kim, T. Cai, C. Richardson, R. Leavitt, and E. Waks, Two-photon interference from a bright single-photon source at telecom wavelengths, Optica 3, 577 (2016).

[16] N. Maring, P. Farrera, K. Kutluer, M. Mazzera, G. Heinze, and H. de Riedmatten, Photonic quantum state transfer between a cold atomic gas and a crystal, Nature (London) 551, 485 (2017).

[17] M. Lettner, M. Mücke, S. Riedl, C. Vo, C. Hahn, S. Baur, J. Bochmann, S. Ritter, S. Dürr, and G. Rempe, Remote Entanglement between a Single Atom and a Bose-Einstein Condensate, Phys. Rev. Lett. 106, 210503 (2011).

[18] N. Kalb, A. A. Reiserer, P. C. Humphreys, J. J. W. Bakermans, S. J. Kamerling, N. H. Nickerson, S. C. Benjamin, D. J. Twitchen, M. Markham, and R. Hanson, Entanglement distillation between solid-state quantum network nodes, Science $\mathbf{3 5 6}$ 928 (2017).

[19] M. Afzelius, C. Simon, H. de Riedmatten, and N. Gisin, Multimode quantum memory based on atomic frequency combs, Phys. Rev. A 79, 052329 (2009).

[20] N. Sinclair, E. Saglamyurek, H. Mallahzadeh, J. A. Slater, M. George, R. Ricken, M. P. Hedges, D. Oblak, C. Simon, W. Sohler et al., Spectral Multiplexing for Scalable Quantum Photonics Using an Atomic Frequency Comb Quantum Memory and Feed-Forward Control, Phys. Rev. Lett. 113, 053603 (2014).

[21] C. Simon, H. de Riedmatten, M. Afzelius, N. Sangouard, H. Zbinden, and N. Gisin, Quantum Repeaters with Photon Pair Sources and Multimode Memories, Phys. Rev. Lett. 98, 190503 (2007).

[22] J. Brendel, N. Gisin, W. Tittel, and H. Zbinden, Pulsed EnergyTime Entangled Twin-Photon Source for Quantum Communication, Phys. Rev. Lett. 82, 2594 (1999).

[23] A. Kuzmich, W. P. Bowen, A. D. Boozer, A. Boca, C. W. Chou, L.-M. Duan, and H. J. Kimble, Generation of nonclassical photon pairs for scalable quantum communication with atomic ensembles, Nature (London) 423, 731 (2003). 
[24] J. F. Clauser, M. A. Horne, A. Shimony, and R. A. Holt, Proposed Experiment to Test Local Hidden-Variable Theories, Phys. Rev. Lett. 23, 880 (1969).

[25] V. Scarani, The device-independent outlook on quantum physics, Acta Phys. Slov. 62, 347 (2012).

[26] C. W. Thiel, N. Sinclair, W. Tittel, and R. L. Cone, $\mathrm{Tm}^{3+}$ : $\mathrm{Y}_{3} \mathrm{Ga}_{5} \mathrm{O}_{12}$ Materials for Spectrally Multiplexed Quantum Memories, Phys. Rev. Lett. 113, 160501 (2014).

[27] M. Afzelius and C. Simon, Impedance-matched cavity quantum memory, Phys. Rev. A 82, 022310 (2010).

[28] M. Rančić, M. P. Hedges, R. L. Ahlefeldt, and M. J. Sellars, Coherence time of over a second in a telecom-compatible quantum memory storage material, Nat. Phys. 14, 50 (2018).

[29] C. O’Brien, N. Lauk, S. Blum, G. Morigi, and M. Fleischhauer, Interfacing Superconducting Qubits and Telecom Photons Via a Rare-Earth-Doped Crystal, Phys. Rev. Lett. 113, 063603 (2014).

[30] X. Fernandez-Gonzalvo, Y.-H. Chen, C. Yin, S. Rogge, and J. J. Longdell, Coherent frequency up-conversion of microwaves to the optical telecommunications band in an Er: YSO crystal, Phys. Rev. A 92, 062313 (2015).
[31] L. Redaelli, G. Bulgarini, S. Dobrovolskiy, S. N. Dorenbos, V. Zwiller, E. Monroy, and J. M. Gérard, Design of broadband high-efficiency superconducting-nanowire single photon detectors, Supercond. Sci. Technol. 29, 065016 (2016).

[32] X. Guo, C.-L. Zou, C. Schuck, H. Jung, R. Cheng and H. $\mathrm{X}$. Tang, Parametric down-conversion photon-pair source on a nanophotonic chip, Light: Sci. Appl. 6, e16249 (2017).

[33] Y. M. Sua, H. Fan, A. Shahverdi, J.-Y. Chen and Y.-P. Huang, Direct generation and detection of quantum correlated photons with $3.2 \mu \mathrm{m}$ wavelength spacing, Sci. Rep. 7, 17494 (2017).

[34] M. Sabooni, Q. Li, S. Kröll, and L. Rippe, Efficient Quantum Memory Using a Weakly Absorbing Sample, Phys. Rev. Lett. 110, 133604 (2013).

[35] T. Böttger, Y. Sun, C. W. Thiel, and R. L. Cone, Spectroscopy and dynamics of $\mathrm{Er}^{3+}: \mathrm{Y}_{2} \mathrm{SiO}_{5}$ at $1.5 \mu \mathrm{m}$, Phys. Rev. B 74, 075107 (2006).

[36] T. Zhong, J. M. Kindem, J. G. Bartholomew, J. Rochman, I. Craiciu, E. Miyazono, M. Bettinelli, E. Cavalli, V. Verma, S. W. Nam et al., Nanophotonic rare-earth quantum memory with optically controlled retrieval, Science 357, 1392 (2017). 